\title{
Exchange and Reconciliation of Clinical Decision Support Outputs across Systems for Coordinated Quality Improvement: Results and Future Direction from an Implementation in a U.S. Population Health Partnership
}

\author{
Junqiao Chen ${ }^{1}$, Aldo Tinoco ${ }^{1}$, Lauren Drinkard ${ }^{1}$, Diane Hunt ${ }^{2}$, Gregory Stevens ${ }^{2}$, John Calhoun ${ }^{2}$, \\ Jason Cassidy ${ }^{1}$, Epson Chiang ${ }^{1}$ and Jesse James ${ }^{1}$
}

${ }^{1}$ Evolent Health, Virginia, 22203, USA

${ }^{2}$ Deaconess Health System, Indiana, 47710, USA

\begin{abstract}
Background: In a population health partnership, shareable clinical decision support (CDS) can reduce service duplication and promote patient wellness by presenting consistent information to all members of a cross-functional, distributed care team. However, existing health IT standards present challenges in the exchange of CDS outputs from multiple systems.

Objectives: To exchange and reconcile CDS outputs across systems in a partnership between an integrated health system (Deaconess Health System) and a population health services organization (Evolent Health).

Methods: We developed a bi-directional HL7-based interface for CDS outputs between Deaconess's Electronic Health Record (EHR) and Evolent's population health services platform (PHSP). The mapping of CDS logic between systems enabled this interface to automatically reconcile inconsistent CDS outputs. Fifteen quality measure (QM)based CDS rules to identify care gaps were selected for this
\end{abstract}

\section{Correspondence to:}

Dr. Jesse James

Evolent Health, 800 N Glebe Road, Suite 500,

Arlington, Virginia, 22203, USA.

E-mail: jjames@evolenthealth.com

\section{Background}

Despite significant investment and effort during the last two decades, progress towards shareable clinical decision support (CDS) has been slow [1]. Existing standards to share CDS artifacts or provide CDS guidance service have not fully meet the need for shareable CDS in a population health partnership. Thus, we developed and implemented a novel bi-directional HL7-based interface to exchange and reconcile CDS outputs. The population health management market is anticipated to have more than fourfold increase in value by 2025, with growth not only in North America but initiative. These care gaps remind Deaconess's clinicians to provide care or documentation necessary for gap closure, and also guide Evolent's care management services.

Results: Two months after launch, Deaconess reconciled 14,040 care gaps from Evolent using data only available in the EHR. Additionally, 1,047 care gaps were resolved due to patient refusal or clinical inappropriateness, and 246 gaps were closed by services or prescriptions provided during clinical encounters.

Conclusions: We implemented an HL7-based interface to exchange and reconcile a large volume of CDS outputs between a health system EHR and a PHSP. Future direction is to standardize the linkage between a CDS rule and its reference QM by universal identifiers and a taxonomy of variations.

\section{Keywords}

Population health; Health information exchange; Quality improvement; Clinical decision support; Health level seven

\section{EJBI 2017; 13(1):35-42}

received: June 11,2017

accepted: July 19, 2017

published: October 10, 2017

the fastest in Asia-Pacific [2]. With this growth, we anticipate a concurrent increase in the integration of Electronic Health Record (EHR) systems and population health services platforms (PHSP), as well as the need to reconcile CDS outputs from each systems.

\subsection{Deaconess Health System-Evolent Health Partnership}

Deaconess Health System (Evansville, Indiana) is an integrated health system of six hospitals, fifteen multispecialty 
community clinics, and a network of community-based providers. Since 2009, Deaconess Health System has used the Epic EHR system (Madison, Wisconsin) with integrated CDS capability. Deaconess Health System has partnered with Evolent Health (Arlington, Virginia), a population health services organization, to assist their transformation from traditional fee-for-service to value-based care. Evolent Health's care management programs are designed to engage patients in collaborative management of their medical, social, and mental health needs, with the goal to optimize wellness and coordinate care. Evolent Health's care managers use its PHSP with integrated CDS capability to identify gaps in care based on quality measures (QM). Care managers and clinicians use the PHSP to review and address care gaps. Because each CDS system has access to different data about patients in the health systems, these two CDS engines together yield a more holistic and comprehensive picture of patients' clinical needs than either system alone.

Deaconess Health System and Evolent Health set out to improve the use of CDS-guided coordination of care. In this partnership, CDS guidance is generated by each of the health IT systems: the EHR system and the PHSP. To ensure the consistency of CDS output presented to users at both Deaconess Health System and Evolent Health, implementation team members examined how to share and reconcile CDS output from both systems.

\subsection{Challenge and Solution}

Several initiatives to accelerate the sharing of CDS have been carried out. For example, the US Health eDecision initiative aims to create standards for CDS artifact sharing and CDS guidance services. CDS artifact sharing is the representation of logic in an electronic format so the receiving application can run it directly; CDS guidance service is the representation of data with a CDS service in exchange for the output of CDS (e.g., care advice) to the requesting system [3].

In the Deaconess-Evolent partnership, the implementation of PHSP alongside the existing EHR system presented challenges that prevented the complete adoption of a traditional approach to share CDS. Integrated health organizations have had systems with locally developed, locally maintained, or commercial-off-the-shelf CDS artifacts deployed in many different representations, based on a variety of data models, and highly contextualized to local settings and workflow [1]. Translating them into a standard format and implementing them across the partnership's care continuum would have taken significant time and effort.

Challenges also appeared in adopting CDS guidance services, which are based on a role-based model in which there is a CDS requester and a responder. In our partnership, both the Deaconess EHR system and the PHSP have had their own CDS engine in place and both have had access to data from different sources. Both system databases contain laboratory result data; however, the Epic EHR system's CDS has had access to provider documentation (e.g., patient refusals for clinical preventive services) and clinical observation data (e.g., vitals and point-of-care lab test results). The PHSP's CDS has had access to medical and pharmacy claims data. Thus, even if two engines were to have the same clinical logic, they might still return different CDS results.

The overarching goal of this project was to exchange and reconcile CDS outputs from both systems, especially when the CDS output varies for a given patient. We developed a solution to address this need: a bi-directional HL7-based interface of CDS outputs supported by quality measureenabled integration and backend reconciliation logic.

The need to align CDS and QM has been widely recognized by HL7 [4], National Quality Forum [5] and US federal agencies [6, 7]. This alignment has become imperative in this current time of US health care reform where providers are being judged by their ability to manage population health defined by QM. The harmonization of QM in our work was partially inspired by the Clinical Decision Support-Quality Measure (CDS-QM) framework pioneered at the University of Utah [8]. In 2014, Kukhareva and colleagues at the University of Utah pioneered a prototype of a standards-based CDS framework aligned with electronic quality measurements [8]. This prototype converted data in a data warehouse into the HL7 virtual medical record (vMR) format and transmitted it to OpenCDS (Figure 1). OpenCDS in turn sent CDS guidance back to the data warehouse.

In this article, we describe the implementation of vital components of the Utah CDS-QM framework in the Deaconess-Evolent partnership. We then discuss the design of the bi-directional, HL7-based interface and the downstream workflow enabled by it.

\section{Methods}

The Deaconess-Evolent Model shares some features of the Utah CDS-QM Framework, but includes additional data sources and supports downstream connectivity with multiple systems, as described below (Figure 2).

\subsection{From Data to CDS Outputs by Evolent}

Firstly, structured data is loaded into Evolent's enterprise data warehouse (EDW) from multiple sources belonging to Deaconess and other entities (health insurers, independent laboratories, etc.). One noted extension to the Utah framework is the addition of pharmacy claims data, which offers important details about fill/refill dates and the actual 


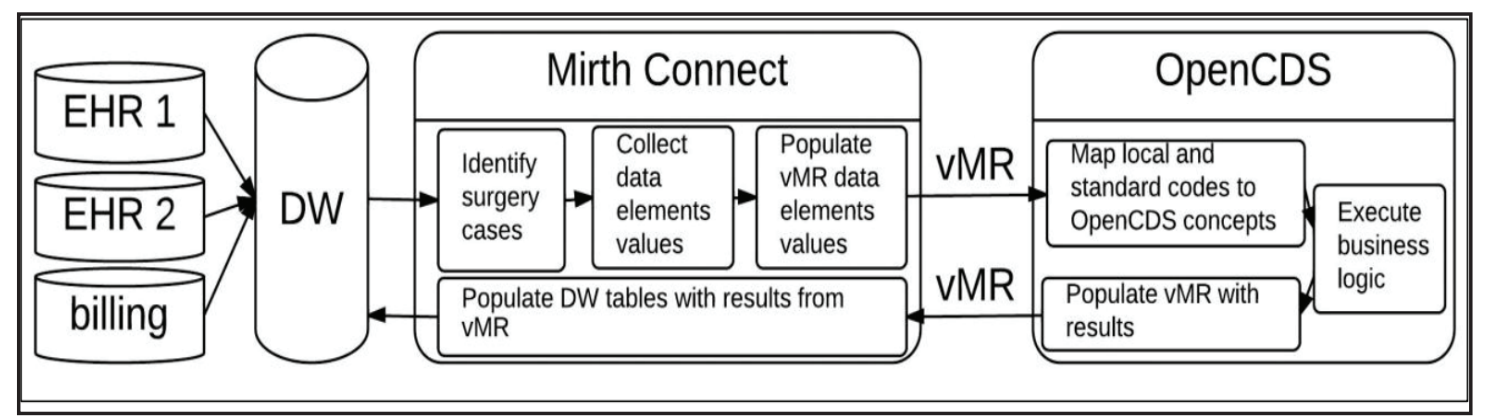

Figure 1: Major systems and processes involved in the Utah CDS-QM approach. Source: [8]. Re-used with permission from the American Medical Informatics Association.

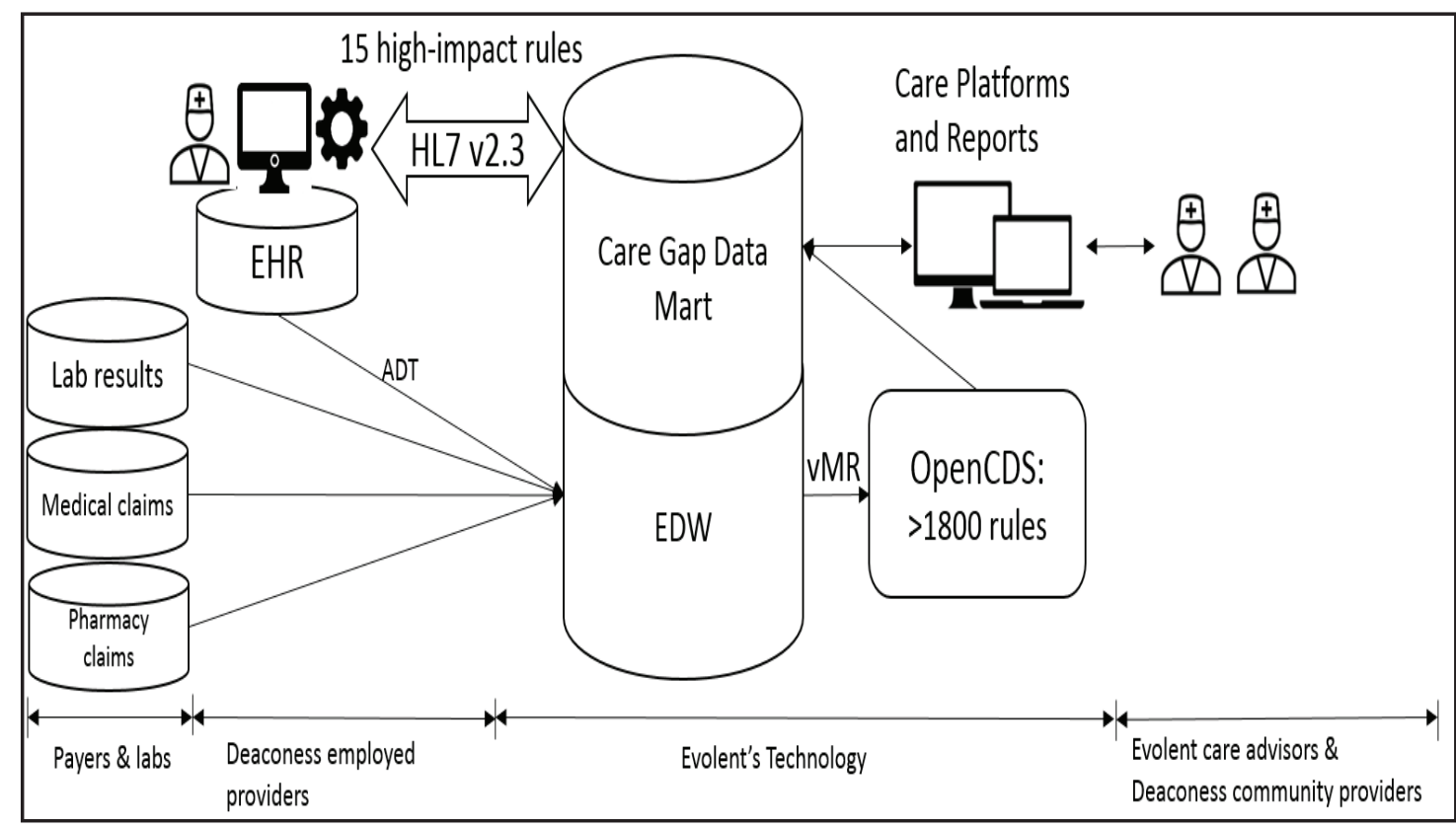

Figure 2: Major systems and processes involved in the Deaconess-Evolent enhanced CDS-QM implementation.

drug dispensed (brand, package, dosage, etc.). Loaded subset of CDS rules was selected for integration into the EHR. data is transformed into vMRs, which is then transmitted Selection criteria included clinical value, current perfomance to an OpenCDS-based rules engine. Evolent Health staff level, physician impactability, technical readiness, and business developed rules to detect care gaps based on QM. The priority. These fifteen "care gap" rules (Table 1) cover chronic relationship between the reference QM, the derived care gap disease management, clinical preventive services, medication decision support rule and their internal unique identifiers are monitoring, and pediatric access to care. For some of these CDS maintained in the rules engine's knowledge base. Output from rules, the need of reconciliation is well-known due to factors like the rules engine is stored in the data warehouse alongside long look-back period for historical data [9].

metadata and other patient-level observations. Evolent's care managers and other users access the CDS outputs via the

Prior to the integration, eleven of these 15 rules existed in PHSP, which also capture interventions performed to close care gaps.

\subsection{Exchange of CDS Outputs}

The decision to build a bi-directional interface between Deaconess and Evolent was the result of a rigorous assessment performed by a cross-functional team. Following a physician-led solution design process, the Deaconess Physician Advisory Council and Outpatient Decision Support Committee met to design the project. A both Evolent and Deaconess platforms with different degrees of variations in logic. Four of these 15 rules heavily rely on pharmacy claims and were only available to Evolent prior the integration. Quality experts from Deaconess and Evolent Health compared the CDS rules from both systems with the corresponding QM specifications. Deconess modified its CDS rules, where necessary, to match logic across systems; thus, inconsistent rule results could be attributed to the differences in data accessible to each system rather than variation in the logic.

Reference Measure: the quality measure that the care gap rule is based upon; NQMC: National Quality Measures 
Table 1: List of care gaps being exchanged and reconciled.

\begin{tabular}{ll}
\hline Care Gap Rules & National Identifiers of the Reference Measures \\
\hline Appropriate Medications for Patients with Asthma & NQMC: 009940, or NQF:0036 \\
Diabetes Care - HbA1c Test & NQMC: 010520, or NQF: 0057 \\
Diabetes Care - Eye Exam & NQMC: 010524, or NQF: 0055 \\
Diabetes Care - Nephropathy Screening & NQMC: 010525, or NQF: 0062 \\
Breast Cancer Screening & NQMC: 009931, or NQF: 2372 \\
Colorectal Cancer Screening & NQMC: 009933, or NQF: 0034 \\
Cervical Cancer Screening & NQMC: 010930, or NQF: 0032 \\
Chlamydia Screening & NQMC: 009934, or NQF: 0033 \\
Annual Flu Vaccination & NQMC: 010565 \& 010566, or NQF: 0039 \& 0040 \\
Pneumococcal Vaccination & NQMC: 010570, or NQF: 0043 \\
Annual Monitoring for Patients on ACE/ARB & NQMC: 010542 Rate 1, or NQF: 2371 Rate 1 \\
Annual Monitoring for Patients on Digoxin & NQMC: 010542 Rate 2, or NQF: 2371 Rate 2 \\
Annual Monitoring for Patients on Diuretics & NQMC: 010542 Rate 3, or NQF: 2371 Rate 3 \\
Well-Child Visits 3 to 6 years-old & NQMC: 010611 , or NQF: 1516 \\
Adolescent Well-Care Visits & NQMC: 010612 \\
\hline
\end{tabular}

Clearinghouse; NQF: National Quality Forum; ACE/ARB: Angiotensin Converting Enzyme inhibitors or Angiotensin Receptor Blockers.

Deaconess maintained a crosswalk between the Evolent CDS rule ID and the clinically equivalent CDS rule in the EHR. Messages sent from the EHR system back to Evolent contain the original ID used by Evolent.

This interface encapsulates care gap messages in HL7 version 2.3 Admission, Discharge, Transfer (ADT) format. HL7 version 2 is used in 95\% of US healthcare organizations [10]. Version 2.3, in particular, is the most widely accepted version by Evolent's partners. Using this standard leverages the existing interfaces in Deaconess's EHR and Evolent's platform. Also, since a significant portion of health systems in the US have installed the same EHR as Deaconess, lessons learned from this implementation will inform future development in other organizations.

There is no existing standard or implementation guide for the use of HL7 v2 segments for care gaps. Due to its extensibility and use for clinical information, we chose to use Observation (OBX) segment capabilities in ADT messages to represent care gaps. Each attribute of a care gap message is represented in one OBX segment, identified by an Observation Identifier. Attributes include: care gap status, Evolent rule ID, open and close dates of the care gap, service provider, and other supporting information. Upon receiving messages from Evolent, Deaconess utilizes the interface engine Cerner OPENLink [11] to process and route messages. Once a message is routed to the EHR, patient identity in the care gap message is matching to the appropriate EHR records by the EHR system.

\subsection{System Reconciliation of Care Gaps}

Automatic reconciliation Figure 3 is triggered when the care gap status is not the same between two systems. The goal is not just reconciling the status, but also prompting the care team to either provide care or data to support the closure of care gaps. The first step is to identify an inconsistent status. Because most care gaps have been tuned to represent the exact same clinical logics, inconsistent status of care gaps could be automatically identified by Deaconess's EHR. When the care gap status is "Open" in Evolent's messages but "Closed" in Deaconess's EHR, supporting data of the gap closure will be automatcially extracted from Deaconess's EHR and sent back to Evolent via the interface. This will update relevant records in Evolent's system.

\subsection{Actions upon Open Care Gaps by Deaconess Providers}

When the care gap status is "Closed" in Evolent but "Open" in Deaconess's EHR, providers are prompted to update the data in EHR to ensure a complete record of care gap closure. When the status is " "Open" in both systems, then a Deaconess provider attempts to offer the clinically appropriate service to close the identified gap or to document reasons why a particular type of service could not be offered/was declined. Providers perform these activites using the Health Maintanence module within the EHR. This is a routinely used module that alerts physicians to various patient needs. Utilizing this module enables a more seamless development and implementation. More importantly, it requires no workflow change to current practices.

\subsection{Actions upon Open Care Gaps by Evolent Care Managers}

Updated care gap statuses are automatically sent back to Evolent every 24 hours and reflected in Evolent's PHSP. Care managers can then address the updated and more 


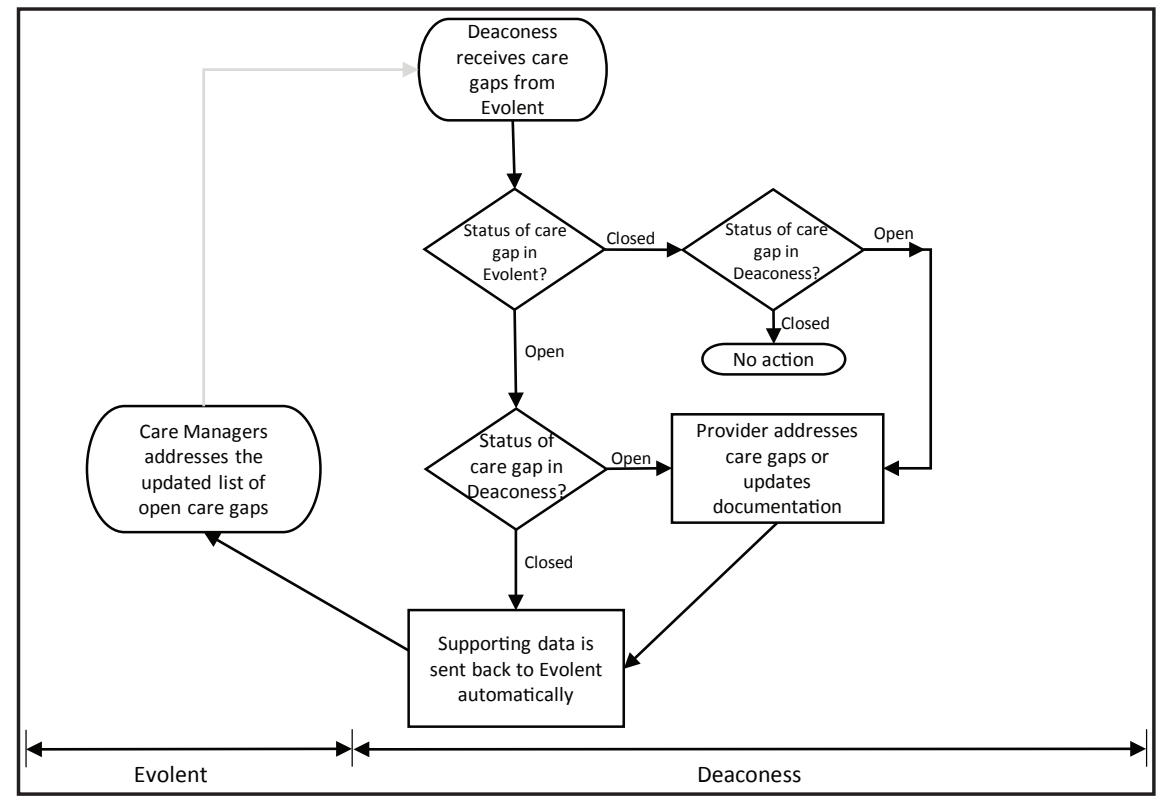

Figure 3: System reconciliation of and care team actions on care gaps.

accurate list of open care gaps by various patient engagement techniques and care management services.

\section{Results}

The bi-directional care gap interface was launched in March 2017. Although there was no change in workflow, provider engagement activities were carried out to inform them of the richer CDS content in the EHR. In the first two months of implementation, Evolent received 15,333 responses from Deaconess (Table 2) via this interface. 14,040 false positive care gaps in Evolent's system were automatically closed by data received from Deaconess via the interface. Additionally, 1,047 responses were either patient refusal or clinical inappropriateness, and 246 gaps were closed by procedures or prescriptions offered during clinical encounters.

Closing a true care gap requires time and resources to engage patients, schedule appointments and provide services. It is encouraging to observe that during the first two months of implementation, Deaconess providers intervened on 1300 care gaps with the help of reconciled CDS outputs. We anticipate this number to increase over time.

\section{Discussion}

Alignment and reconciliation of CDS "are developing areas in informatics that will need continued investigation

Table 2: Number of care gaps received by evolent from deaconess categorized by closure reasons; March to May, 2017.

\begin{tabular}{llll}
\hline $\begin{array}{l}\text { Closed by } \\
\text { offering care }\end{array}$ & $\begin{array}{l}\text { Patient } \\
\text { refused or not } \\
\text { Appropriate }\end{array}$ & $\begin{array}{l}\text { Closed by data } \\
\text { only available } \\
\text { in EHR }\end{array}$ & Total \\
\hline 246 & 1047 & 14040 & 15333 \\
\hline
\end{tabular}

as CDS implementations increase" [12]. Benefits include the reduction of waste (e.g., human effort and duplication of services), provider dissatisfaction, and confusion (e.g., basing outreach to patients on outdated information). Thus it enables a consistent user experience and increases the coordination of care across systems [13].

Evolent Health utilizes an open-source CDS-QM framework to turn QM into actionable CDS outputs to alert providers of gaps in patients' care. To reconcile these outputs with Deaconess Health System's practice EHR, an HL7-based bi-directional interface was built and implemented for 15 care gap rules selected by physician leaders. Due to the physicianled mapping of CDS logics between systems prior the implementation of the interface, care gaps were automatically reconciled using data that was previously inaccessible to Evolent Health.

This work presents a novel solution to electronically exchange and reconcile the output of different CDS systems via existing interface rather than build new interfaces to exchange raw observation data between the underlying data warehouses (e.g., vital sign values, lab results, medications, diagnoses, and procedures). A traditional approach to ensure that different CDS systems generate the same output would be to ensure that different systems have the same data input available to the same logic. However, adding additional clinical data exchange interfaces incurs significant expense. A survey of 125 organizations found that financial cost of building interfaces is the number one challenge to interoperability. In all, 112 organizations had to construct multiple interfaces, and 18 reported having to construct more than 25 interfaces [14]. This is burdensome work with substantial cost from developing and mapping multiple 
interfaces, terminology mapping, quality assurance, and maintenance. Research has shown that only one-fifth of US hospitals engage in key domains of interoperability [15], and therefore significant care coordination gaps exist due to the lack of interoperability between health IT systems [16]. The fact that patient data is represented in various schemas and coded in different vocabularies across data sources is not only barrier to building a raw data interface, but also important challenges to clinical knowledge sharing. This is referred to as the "curly braces problem" in the space of HL7 Arden Syntax $[17,18]$. While our approach uses information that is less granular than raw clinical observation data, it enables successful exchange and reconciliation of CDS outputs that add to the patient's clinical picture. It presents meaningful information for the end users.

We acknowledge three limitations to our approach to this implementation. First, the lack of a mechanism to reference a universal identifier for each care gap required time-consuming manual review of clinical logic between two organizations. This is a barrier to achieving scale when increasing the number of healthcare organizations or CDS rules in this exchange. Second, although HL7 v2 has wide adoption and fulfilled the needs of the project, it requires a significant amount of customization to fully achieve interoperability in each unique context. Additionally, even with all the necessary leg work, the message structure still tends to be complex, flat and delimited [19]. Examining the use of HL7 version 3 or Fast Healthcare Interoperability Resources (FHIR) standards is beyond the scope of this initial project, but the use of FHIR and other application programming interface-based exchange of CDS output should be discussed and explored by the industry. Finally, this interface does not always capture sufficient detail to with other related use cases that require data at the observation level, such as the regulatory requirement of QM reporting. Our specific use case is to deliver care gaps within a workflow tool to proactively and prospectively improve the quality of care; this is certainly related to but not the same as QM reporting programs. Additional configuration (e.g. mandating physician's detailed input of all required data elements and attestation) will be needed for other use cases.

\section{$5 \quad$ Future Work}

Future research is needed to support the sharing of CDS output between health IT systems. Care gap detection logic is derived from either QM or from the clinical practice guidelines that the QM are based on. How can systems consistently manage the relationships between CDS rules and the reference QM from which it was derived? There are several situations in which a CDS content developer chooses to modify rule logic: for example, to address local workflow needs, to adapt to unique data models, and to allow locally defined data values. How should rule developers and implementers classify variations introduced in CDS rules from the reference QM and from other similar CDS rules? Since CDS output is used in a variety of settings across the continuum of care, what attributes are required to inform or proscribe interventions to close care gaps that are relevant to the setting of their use (e.g., clinical setting, care management setting, or directed to patients themselves)? Findings from research in these areas will inform the development of exchange standards to streamline the implementation of CDS output deployed across the various settings of care, enabling proactive population health management.

\subsection{Universal Quality Measure Identifier System}

This implementation highlights the urgency of establishing a formal identifier system in HL7 for QM and derivative CDS logic. In our example, a challenge rests within the receiving entity (Deaconess's EHR) to understand, process and correctly display care gaps in the user interface. It required a group of experts to manually review the care gap logic from the sending entity (Evolent), and where applicable, map to or modify existing internal rules within the EHR. This is not a new challenge, as Greenes and colleagues [20] demonstrated a similar issue in a previous informatics project (Morningside Initiative, 2008).

The most direct solution is to develop a mechanism to reference a centralized, standardized and authoritative identifier system for QM, which could enable the identification of reference measures in CDS-QM related development [21]. A major barrier is that there is no single universal identifier system for QM and CDS in the United States. For example, the National Quality Forum (NQF) has a numbered inventory of 1,086 measures [22], and the National Quality Measures Clearinghouse (NQMC) has a numbered inventory of 2,342 measures [23]. The distributed nature of the U.S. healthcare industry leaves no single entity responsible for maintaining a complete set of QM publicly available. Furthermore, the existing major repositories do not support a crosswalk between their identifiers. Internationally, this may be less a barrier in countries with centralized healthcare systems. China, for example, has been maintaining a single Chinese Healthcare Quality Indicators System (CHQIS) since 2009 with more than 5,000 numbered indicators in scientific hierarchies [24].

The need to unambiguously reference a QM has been recognized by HL7 in its recent development of FHIR. In the Measure resource of FHIR [25], there is an "Identifier system" element, which refers to one or more measure development bodies like NQF by a Uniform Resource Locator (URL). Currently there is no official identifier system established in FHIR yet, but it is understood that work is in progress (Bryn Rhodes, personal communication, 2017 May 23). An excerpt from FHIR website is shown below Figure 4. A similar approach might be applied to HL7 v2 in its extendable segments as well. 


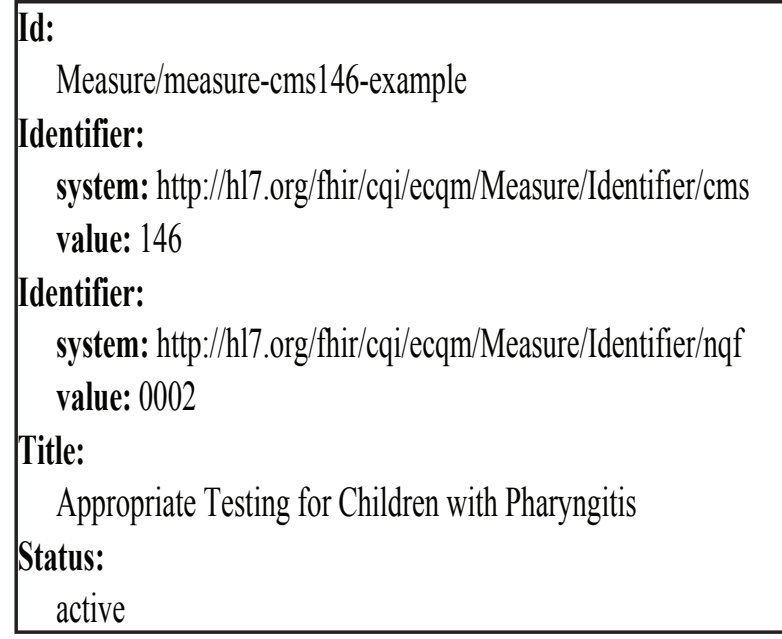

Figure 4: "Identifier System" example from FHIR. Source: [26].

\subsection{Taxonomy to Classify Variations from Reference Measure}

If the above reference system is in place, then the next question is how to represent variations from the "reference measure" in a way that computers can interpret. This is very difficult due to the complexity and variabilitvy in changes made to the reference measure across systems.

Yauch and colleagues drafted a taxonomy to "capture the types of variations introduced in CDS rules as they are deployed to a specific setting" [27]. The first level of its taxa includes Threshold Factor, Timing Factor, Setting Factor, Event, Contraindicating Factor, Intervention, CDS Destination, Event Inducing Action, and CDS Realization Method. Capturing information like this will add to the complexity of initial implementation. The benefit of doing this could be realized as CDS output is increasingly exchanged. As an industry, we should achieve consensus on a first, core set of variation types, and then build upon this initial set to expand the taxonomy, covering all necessary variations. The allowed degree and type of variation should be carefully examined. It is recommended that if the variation in CDS rule greatly differs from the reference QM, it may be better to work with the QM steward to create a new measure corresponding to that CDS rule [21].

\subsection{Contexts-Aware Attributes of a Care Gap}

While the above two topics focus on the semantic exchange and reconciliation of care gaps, we must also consider what supporting information is necessary to selectively target CDS output to the right recipients based on their respective roles, their scope of practice, and per the partnership agreements made between the health care organizations involved in CDS output exchange.

Care managers who currently use the PHSP suggested the creation of context awareness attributes - "action by" and "action type" - to indicate the type of providers who should perform the type of action necessary to close a care gap. Take the Diabetes Eye Exam care gap as an example. It should have two sets of attributes: first, [action by] = "ophthalmologist or optometrist" and [action type] = "perform a retinal exam", and second, [action by] = "non-ophthalmologist or optometrist" and [action type] = "refer this patient to ophthalmologist or optometrist". Depending on the specialty of the provider offering the service ("context-aware"), one of the "action type" values will be shown. A standard, core set of attributes can be developed after the implementation of additional care gap rules. Broad participation in industry is essential in this adventure.

\section{Conclusion}

We expanded an HL7-based CDS and QM framework and applies it in a population health partnership. A novel bi-directional HL7-based care gap interface is built to exchange and reconcile CDS outputs ("care gaps") between a commercial EHR and a PHSP. Preliminary data shows that the interface has automatically reconciled false care gap alerts across organizations in the short time it has been implemented. This is an effective, HL7 standards-enabled, pragmatic implementation in a complex distributed care landscape. Future work is proposed to automate semantic mapping of CDS outputs between organizations by both a universal identifier system and a taxonomy of variations, and to standardize contextual attributes to better inform responses to care gaps.

\section{Acknowledgements}

We thank all the colleagues who contributed to the design, development, implementation and continuous maintenance of this interface. We thank Mr. Nicholas Dus, Mr. Rami ElGawly, Ms. Rahel Ephrem, Ms. Connie Weber, Mr. Milesh Patel and Mr. Robert Goska, for their insightful comments on earlier versions of the manuscript.

\section{References}

[1] Greenes RA, Peleg M, Rector A, Osheroff J. Reusable Knowledge for Best Clinical Practices: Why We Have Difficulty Sharing And What We Can Do. InMedinfo 2013;1237.

[2] Population Health Management (PHM) Market 2014 - 2025. Grand View Research, 2016.

[3] Fridsma, D. Two Significant S\&I Framework Milestones: the Health eDecision Initiative Close-Out and Clinical Quality Framework Launch. 2014. Available from: https://www.healthit. gov/buzz-blog/electronic-health-and-medical-records/ interoperability-electronic-health-and-medical-records/ standards-interoperability-framework-milestones-healthedecision-initiative-closeout-clinical-quality-frameworklaunch 
[4] Health Level Seven International, Inc. Quality Improvement Core (QI-Core) Implementation Guide [Internet]. Ann Arbor, Michigan: Health Level Seven International; 2016 May 31 [cited 2017 May 23]. Available from: http://www.hl7.org/fhir/ current/qicore/

[5] Driving Quality and Performance Measurement - A Foundation for Clinical Decision Support: A Consensus Report. National Quality Forum. 2010.

[6] Clinical Decision Support: More than Just 'Alerts'. Centers for Medicare and Medicaid Services, 2014.

[7] Berner, E. S. Clinical Decision Support Systems: State of the Art. Agency for Healthcare Research and Quality; 2009; Report No.: 09-0069-EF

[8] Kukhareva PV, Kawamoto K, Shields DE, Barfuss DT, Halley AM, Tippetts TJ, et al. Clinical Decision Support-based Quality Measurement (CDS-QM) Framework: Prototype Implementation, Evaluation, and Future Directions. InAMIA Annual Symposium Proceedings.American Medical Informatics Association, 2014.

[9] Wagholikar K, Sohn S, Wu S, Kaggal V, Buehler S, Greenes RA, et al. Workflow-based Data Reconciliation for Clinical Decision Support: Case of Colorectal Cancer Screening and Surveillance. AMIA Summits on Translational Science Proceedings. 2013 March 18-22; San Francisco, USA. Washington DC; 2013.

[10] Health Level Seven International. HL7 Version 2 Product Suite [Internet]. Ann Arbor, Michigan: Health Level Seven International; 2017 March 21 [cited 2017 Jun 5]. Available from: http://www.hl7.org/implement/standards/product_ brief.cfm?product_id $=185$

[11] Cerner OPENLink [Internet]. Cerner Corporation. 2009. Available from: http://www.cerner.com/page. aspx?pageid $=17179878387 \&$ libID $=17179878602$

[12] Tso GJ, Tu SW, Oshiro C, Martins S, Ashcraft M, Yuen KW, et al. Automating Guidelines for Clinical Decision Support: Knowledge Engineering and Implementation. AMIA Annual Symposium Proceeding; 2016 Nov 12-16; Chicago, USA. Washington DC: American Medical Informatics Association; 2016.

[13] Asanjan SMH. Consolidation of CDA-Based Documents from Multiple Sources: A Modular Approach [dissertation]. 2016.

[14] Hall SD. Interoperability remains a barrier for health data exchange organizations [Internet]. In: FierceHealthcare [Internet]. 2014. Available from http://www.fiercehealthcare. com/it/interoperability-remains-a-barrier-for-health-dataexchange-organizations
[15] Holmgren AJ, Patel V, Charles D, Adler-Milstein J. US Hospital Engagement in Core Domains of Interoperability. Am J of Manag Care. 2016; 22: e395-e402.

[16] Samal L, Dykes PC, Greenberg JO, Hasan O, Venkatesh AK, Volk LA, Bates DW. Care coordination gaps due to lack of interoperability in the United States: a qualitative study and literature review. BMC Health Serv Res. 2016; 16:143.

[17] Health Level Seven International, Inc. HL7 Cross-Paradigm Specification: Clinical Quality Language, Release 1 [Internet]. Ann Arbor, Michigan: Health Level Seven International; 2016 July [cited 2017 Jun 8]. Available from: http://www.hl7.org/ implement/standards/product_brief.cfm?product_id $=400$

[18] Greenes RA. Clinical Decision Support: The Road to Broad Adoption. 2nd ed. Amsterdam: Academic Press.2014; 930.

[19] Musal S. The Differences Between HL7 and FHIR (and Why You Should Care) [Internet]. 2016 In: CloudMine Blog [Internet]. 2016. Available from: http:// blog.cloudmineinc.com/the-differences-between-hl7-andfhir-and-why-you-should-care

[20] Greenes R, Bloomrosen M, Brown-Connolly NE, Curtis C, Detmer DE, Enberg R, et al. The morningside initiative: collaborative development of a knowledge repository to accelerate adoption of clinical decision support. Open Med Inform J. 2010; 4: 278-90.

[21] National Quality Forum. Variation in Measure Specifications: Sources and Mirigation Strategies (Final Report). 2016; Report No.: (ISBN) 978-1-68248-032-8. Contract No.: HHSM-5002012-00009I.

[22] National Quality Forum. Quality Positioning System [Internet]. 2017. Available from: http://www.qualityforum. org/Qps/QpsTool.aspx

[23] National Quality Measures Clearinghouse [Internet].Agency for Healthcare Research and Quality. 2017. Available from: https://qualitymeasures.ahrq.gov/

[24] Zhao M, Liang, M, Yu R, Jian W, Zhang L, Ma W. Designing and application on CHQIS medical quality evaluation system. Chinese Hospitals. 2009; 13: 1-7.

[25] Health Level Seven International. Resource Measure Content [Internet]. Ann Arbor, Michigan: Health Level Seven International; 2017 Jun [cited 2017 Jun 9]. Available from: http://build.fhir.org/measure.html

[26] Health Level Seven International. FHIR Example Instance [Internet]. Ann Arbor, Michigan: Health Level Seven International; 2017 Jun [cited 2017 Jun 9]. Available from: http://build.fhir.org/measure-cms146example.xml

[27] Yauch D, Kum P, Tu S, Haug P, Hulse N, Fry E, et al. Development of a Taxonomy of Setting-Specific Factors for Adaptation of Clinical Decision Support Rules. AMIA Annual Symposium, American Medical Informatics Association, 2012. 